\title{
Pressure and Variational Principle for Random Ising Model
}

\section{F. Ledrappier}

Université Pierre et Marie Curie, Laboratoire de Calcul Probabilités, F-75230 Paris, France

\begin{abstract}
An Ising model traditionally is a model for a repartition of spins on a lattice. Griffiths and Lebowitz ([3.5]) have considered distributions of spins which can occur only on some randomly prescribed sites-Edwards and Anderson have introduced models where the interaction was random $([6,7])$. In both cases, the formalism of statistical mechanics reduces mainly to a relativised variational principle, which has been proved recently by Walters and the author [1]. In this note, we show how that reduction works and formulate the corresponding results on an example of either model.
\end{abstract}

\section{Notations and Results}

Let $Y=\{0,1\}^{Z^{d}}, X=\{0,+1,-1\}^{Z^{d}}$ be the sets of configurations of particles (respectively of particles with a spin) on a lattice $Z^{d}$, Let $\pi: X \rightarrow Y$ denote the natural map such that $(\pi(x))_{s}=\left|x_{s}\right|$ for $s$ in $Z^{d}$, $\tau_{s}$ the shift transformations on $X$ and $Y, \Lambda_{n}$ the positive cube of side $n$ containing the point $(0,0, \ldots, 0)$ of $Z^{d}$. A point $y$ is said generic for an invariant measure $v$ on $Y$ if the measures $\frac{1}{n^{d}} \sum_{s \in \Lambda_{n}} \delta_{\tau_{s} y}$ converge towards the measure $v\left(\delta_{z}\right.$ denotes the Dirac measure at the point $\left.z\right)$.

Let $J, h$ be real numbers. For $x$ in $X$ with $x_{s}=0$ except for a finite number of $s$, define:

$$
U(x)=\sum_{s \in \mathbf{Z}^{d}} h x_{s}+\sum_{\substack{s, t \in \mathbf{Z}^{d} \\|s-t|=1}} J x_{s} x_{t},
$$

where $|s|=\sum_{i}\left|s_{i}\right|$ if $s=\left(s_{i}, i=1, \ldots, d\right)$.

For any finite subset $\Lambda$ of $Z^{d}$ and any $y$ in $Y$ let us consider the partition function of the box $\Lambda$ above $y Z_{\Lambda}(y)$ :

$$
Z_{\Lambda}(y)=\sum \exp (-U(x))
$$

where the summation is made over the set of $x$ such that $\left|x_{s}\right|=y_{s}$ for $s$ in $A, x_{s}=0$ elsewhere. Let $M(X, \tau)$ denote the set of invariant probability measures on $X$. 
For $\mu$ in $M(X, \tau)$ and $A$ a finite measurable partition of $X$, we consider $H(\mu, A)$ the mean entropy of $A$, and define the entropy $h(\mu)$ by: $h(\mu)=\sup _{A} H(\mu, A)$. Let us define also the conditional entropy $h(\mu / Y)$ by:

$h(\mu / Y)=\sup _{A} \inf _{B} H(\mu, A)-H\left(\mu, \pi^{-1}(B)\right)$, where $A$ (resp. $\left.B\right)$ is a partition of $X$ [resp. a partition of $Y$ with $\pi^{-1}(B)$ coarser than $A$ ]. If $h\left(\mu \circ \bar{\pi}^{-1}\right)$ is finite, we have the following formula:

$$
h(\mu / Y)=h(\mu)-h\left(\mu \cdot \pi^{-1}\right) \quad(\text { see }[2]) .
$$

Theorem 1. If $y$ is generic for some measure $v$ then the sequence $\frac{1}{n^{d}} \log Z_{\Lambda_{n}}(y)$ converges as $n$ goes to infinity towards a number $P_{v}$ called the pressure above $v$; the pressure above $v$ satisfies the following variational principle:

$$
P_{v}=\max _{\substack{\mu \in M(X, \tau) \\ \mu \circ \pi^{-1}=v}} h(\mu)-h(v)+\int a(x) d \mu
$$

where $a(x)=-h x_{0}-\frac{J}{2} \sum_{|s|=1} x_{0} x_{s}$

Note that if $v$ is ergodic almost every point $y$ is generic.

Let $S$ be the set of pairs of neighbours in $\boldsymbol{Z}^{d}$; the translations of $\boldsymbol{Z}^{d}$ act naturally on $S$.

Let $\mathbb{R}$ denote the real line and fix $y^{\prime}$ in $Y^{\prime}=\mathbb{R}^{S}$. We can define by the usual formulas the partition functions $P_{\Lambda}\left(y^{\prime}\right)$ of a finite box $\Lambda$ corresponding to the interaction $J_{i, j}$

$$
J_{i, j}=y_{\{i, j\}} \text { if } i \text { and } j \text { are neighbours , }
$$

on the space $X^{\prime}=\{-1,+1\}^{Z^{d}}$ of spins on the lattice $Z^{d}$.

Theorem 2. Let $v$ be a $Z^{d}$-invariant, ergodic probability measure on $Y^{\prime}$, such that $\sup _{t} \int\left|y_{t}\right| d v<\infty$.

The limit $\lim _{n \rightarrow \infty} \frac{1}{n^{d}} \log P_{\Lambda_{n}}\left(y^{\prime}\right)$ exists for almost every $y^{\prime}$ and satisfies a variational principle. (See Vuillermot [8] for a close result when the $y_{i, j}$ are independent.)

\section{Proof of Theorem 1}

We recall first the notation and results from [1], in a suitable form.

Let $X, Y$ compact metric spaces, $\pi: X \rightarrow Y$ a surjection and a $Z^{d}$ action on $X$ and $Y$ which commutes with $\pi$. Let $\varepsilon>0, n$ integer be given, $d$ denote a distance on $X$.

A set $E$ in $X$ is said $(n, \varepsilon)$ separated if for any $x_{1} \neq x_{2}$ in $E, \sup _{i \in A_{n}} d\left(\tau_{i} x_{1}, \tau_{i} x_{2}\right)$ is greater than $\varepsilon$. For $f$ continuous function on $X, y$ in $Y$, we define:

$$
P_{n}(\tau, f, y, \varepsilon)=\sup _{E} \sum_{x \in E} \exp \left(\sum_{i \in \Lambda_{n}} f\left(\tau_{i} x\right)\right)
$$

where the sup is taken over the $(n, \varepsilon)$ separated sets $E$ with $\pi(x)=y$ for every $x$ in $E$

$$
p(\tau, f, y)=\lim _{\varepsilon \rightarrow 0} \limsup _{n \rightarrow \infty} \frac{1}{n^{d}} \log p_{\dot{n}}(\tau, f, y, \varepsilon) .
$$


Theorem 3 ([1], Proposition 3.5). For any invariant measure $\mu$ on $X$, we have:

$$
h(\mu / Y)+\mu(f) \leqq \sup _{\varepsilon} \limsup _{n \rightarrow \infty} \int \frac{1}{n^{d}} \log p_{n}(\tau, f, y, \varepsilon) d \mu \circ \pi^{-1}(y) .
$$

Remark. Actually Proposition 3.5 in [1] is stated with $\int p(\tau, f, y) d \mu \circ \pi^{-1}(y)$ instead of sup $\lim \sup . .$. . But this stronger result is also true by not applying Fatou's lemma at the end the proof of the Proposition 3.5.

Theorem 4 ([1], Proposition 3.6). If $y$ is generic for some measure $v$ and $\varepsilon$ positive, there exists an invariant measure on $X$ such that $\mu \circ \pi^{-1}=v$ and

$$
h(\mu / Y)+\mu(f) \geqq \limsup _{n \rightarrow \infty} \frac{1}{n^{d}} \log p_{n}(\tau, f, y, \varepsilon) .
$$

As the entropy $h(\mu / Y)$ is upper semi-continuous on the space $M(X, \tau)$ and as the set of measures which projects onto $v$ is a closed subset of $M(X, \tau)$ there exists a measure $\mu_{0}$ such that $\mu_{0} \circ \pi^{-1}=v$ and:

$$
h\left(\mu_{0} / Y\right)+\mu_{0}(f)=\sup _{\substack{\mu \in M(X, \tau) \\ \mu \circ \pi^{-1}=v}} h(\mu / Y)+\mu(f) .
$$

Therefore Theorem 1 will be proved when we shall have shown the following inequalities :

$$
\text { (*) } \begin{aligned}
\lim \sup _{n} \frac{1}{n^{d}} \log Z_{\Lambda_{n}}(y) & \leqq \sup _{\substack{\mu \in M(X, \tau) \\
\mu \circ \pi^{-1}=v}} h(\mu / Y)+\mu(a) \\
& \leqq \lim _{n} \inf \frac{1}{n^{d}} \log Z_{\Lambda_{n}}(y)
\end{aligned}
$$

as soon as $y$ is generic for $v$.

We prove these relations with two lemmas:

Let us take on $X$ the distance $\delta$ defined by $\delta\left(x^{1}, x^{2}\right)=\alpha^{k}$, where $0<\alpha<1$ and $k$ is the smallest positive integer such that there exists $s=\left(s_{1}, \ldots, s_{d}\right)$ in $Z^{d}$ with $\sup _{J} s_{j}=k$ and $x_{s}^{1} \neq x_{s}^{2}$.

Lemma 5. For any $y$ in $Y, \varepsilon>0, \frac{1}{n^{d}} \log Z_{\Lambda_{n}}(y) \leqq \frac{1}{n^{d}} \log p_{n}(\tau, a, y, \varepsilon)+\frac{2^{d} J}{n}$, for any $y$ in $Y, \varepsilon>0$, there exists $m$ such that:

$$
\frac{1}{n^{d}} \log p_{n}(\tau, a, y, \varepsilon) \leqq \frac{1}{n^{d}} \log Z_{\Lambda_{n}}(y)+\frac{2^{d} J}{n}+\frac{(2 m)^{d} \log 2}{n} .
$$

Proof. Take $\varepsilon>\alpha$. A set $E$ is $(n, \varepsilon)$ separated if and only if any two different points in $E$ have some different coordinate in $\Lambda_{n}$. So the set of $x$ such that $\left|x_{s}\right|=y_{s}$ for $s$ in $\Lambda_{n}$, $x_{s}=0$ elsewhere is $(n, \varepsilon)$ separated and we may write, by estimation of the boundary effect

$$
Z_{\Lambda_{n}}(y) \leqq p_{n}(\tau, a, y, \varepsilon) \cdot \exp \left(2^{d} n^{d-1} J\right) .
$$

On the other hand for any $\varepsilon$ there exists $m$ such that $\varepsilon>\alpha^{m}$ and so if a set $E$ is $(n, \varepsilon)$ separated any two different points in $E$ have some different coordinate $s$ with $-m \leqq s_{j}<n+m$. If $z$ is some point with $\left|z_{s}\right|=y_{s}$ for $s$ in $\Lambda_{n}, z_{s}=0$ elsewhere, there are 
at most $2^{(2 m)^{d} n^{d-1}}$ different points in $E$ with $x_{s}=z_{s}$ for all $s$ in $\Lambda_{n}$. For any $(n, \varepsilon)$ separated set $E$ in $\pi^{-1}(y)$ we have:

$$
\sum_{x \in E} \exp \left(\sum_{i \in \Lambda_{n}} a\left(\tau_{i} x\right)\right) \leqq 2^{(2 m)^{d} n^{d-1}} \cdot \exp \left(2^{d} n^{d-1} J\right) \cdot Z_{\Lambda_{n}}(y), \quad \text { q.e.d. }
$$

Corollary 6. For any $y$ in $Y$, any measure $v$ on $Y$ :

$$
\begin{aligned}
& \lim _{n} \sup \frac{1}{n^{d}} \log Z_{\Lambda_{n}}(y)=p(\tau, a, y), \\
& \lim \sup _{n} \frac{1}{n^{d}} \int \log Z_{\Lambda_{n}}(y) d v(y)=\lim _{\varepsilon \rightarrow 0} \lim \sup _{n} \frac{1}{n^{d}} \int \log p_{n}(\tau, a, y, \varepsilon) d v(y) .
\end{aligned}
$$

Lemma 7. If $y$ is generic for some measure $v$, we have:

$$
\lim _{n} \sup \frac{1}{n^{d}} \int \log Z_{\Lambda_{n}}(y) d v(y) \leqq \lim _{n} \inf \frac{1}{n^{d}} \log Z_{\Lambda_{n}}(y) \text {. }
$$

Proof. Let us take $m>n, j$ in $\Lambda_{n}$. The box $\Lambda_{m}$ is made of disjoint boxes $\Lambda_{n}+j+n s$, where $n s=\left(n s_{1}, \ldots, n s_{d}\right), s_{i}$ is a positive integer smaller than $\frac{m}{n}-1$, and of less that $(2 n)^{d} m^{d-1}$ other points.

There are less than $\left(\frac{m}{n}\right)^{d} 2^{d} n^{d-1}$ points in the boundaries of the small $\Lambda_{n}+j+n s$ boxes. Therefore we may write:

$$
\begin{aligned}
\log Z_{\Lambda_{m}}(y) \geqq & \sum_{s, 0 \leqq s_{l}<\frac{m}{n}-1} \log Z_{\Lambda_{n}}\left(\tau_{n s+j} y\right) \\
& -J 2^{d} n^{d-1}\left(\frac{m}{n}\right)^{d}-(h+2 J)(2 n)^{d} m^{d-1} .
\end{aligned}
$$

Averaging over all $j$ in $\Lambda_{n}$, dividing by $m^{d}$ and taking $\lim _{m}$ inf, we get by the generiticity of $y$ :

$$
\liminf _{m} \frac{1}{m^{d}} \log Z_{\Lambda_{m}}(y) \geqq \int \frac{1}{n^{d}} \log Z_{\Lambda_{n}}(y) d v(y)-J \frac{2 d}{n} .
$$

The lemma follows by taking lim sup.

The inequalities $(*)$ are proved by comparison of Theorems 3 and 4, Corollary 6 , and Lemma 7.

\section{Proof of Theorem 2}

Let us choose a sequence of continuous real functions $g_{k}$ on $\boldsymbol{R}$ with compact support such that

$$
\delta_{k}=\sup _{t} \int\left|g_{k}\left(y_{t}\right)-y_{t}\right| d v \text { goes to } 0 \text { as } k \text { goes }
$$

to infinity.

Let $P_{\Lambda}^{k}\left(y^{\prime}\right)$ be the partition function on $X^{\prime}$ corresponding to the interaction $J_{i, j}^{k}$ : $J_{i, j}^{k}=g_{k}\left(y_{\{i, j\}}\right)$ if $i$ and $j$ are neighbours . 
Let $a_{k}$ and $a$ be real continuous functions on the product space $Y^{\prime} \times X^{\prime}$ defined by

$$
\begin{aligned}
& a_{k}\left(y^{\prime}, x^{\prime}\right)=-\frac{1}{2 d} \sum_{|s|=1} g_{k}\left(y_{\{0, s\}}\right) x_{0} x_{s} \\
& a\left(y^{\prime}, x^{\prime}\right)=-\frac{1}{2 d} \sum_{|s|=1} y_{\{0, s\}} x_{0} x_{s} .
\end{aligned}
$$

For any $k$, the following lemma is got by considering $Y^{\prime}$ as a factor of $Y^{\prime} \times X^{\prime}$.

Lemma 8. For $v$ almost every $y^{\prime}$, we have

$$
\lim _{n \rightarrow \infty} \frac{1}{n^{d}} \log P_{x o}^{k}\left(y^{\prime}\right)=\max _{\substack{\mu \in M\left(Y^{\prime} \times X^{\prime}, \tau\right) \\ \mu \circ \pi^{-1}=v}} h\left(\mu \mid Y^{\prime}\right)+\int a_{k} d \mu .
$$

Let us consider the compact spaces $\overline{\boldsymbol{R}}=\boldsymbol{R} \cup\{\infty\}$ and $\bar{Y}^{\prime}=\overline{\boldsymbol{R}}^{S}$. The space $Y^{\prime}$ is naturally continuously imbedded in $\bar{Y}^{\prime}$, the function $a_{k}$ is the restriction to $Y^{\prime} \times X^{\prime}$ of a continuous function $\overline{a_{k}}$ on $\bar{Y}^{\prime} \times X^{\prime}$, the measure $v$ is the measure induced on the invariant set $Y^{\prime} \times X^{\prime}$ by an invariant ergodic measure $\bar{v}$ on $\bar{Y}^{\prime} \times X^{\prime}$.

We get then by the same estimations as in $\S 2$ : If $y$ is generic for $\bar{v}$, we have:

$$
\lim _{n \rightarrow \infty} \frac{1}{n^{d}} \log P_{\Lambda_{n}}^{k}(y)=\max _{\substack{\bar{\mu} \in M\left(\bar{Y}^{\prime} \times X^{\prime}, \tau\right) \\ \bar{\mu} \circ \pi^{-1}=\bar{v}}} h(\bar{\mu} \mid \bar{Y})+\int \bar{a}_{k} d \bar{\mu} .
$$

Lemma 8 follows by observing that almost every $y^{\prime}$ in $Y^{\prime}$ is generic for $\bar{v}$ and that measures on $\bar{Y}^{\prime} \times X^{\prime}$ which projects onto $\bar{v}$ are actually carried by $Y^{\prime} \times X^{\prime}$.

We also have the following uniform approximations:

Lemma 9. For any measure $\mu$ such that $\mu \circ \pi^{-1}=v$,

$$
\left|\int a_{k} d \mu-\int a d \mu\right| \leqq \delta_{k}
$$

obvious.

Lemma 10. The sequence of functions on $Y^{\prime}, s_{k}(y)$

$$
s_{k}(y)=\sup _{n} \frac{1}{n^{d}}\left|\log P_{\Lambda_{n}}(y)-\log P_{\Lambda_{n}}^{k}(y)\right|
$$

converges to zero in probability (i.e. for any $\alpha v\left(s_{k} \geqq \alpha\right) \rightarrow 0$ ).

Proof of Lemma 10. We have for any $y$ and any $n$

$$
\left|\log P_{\Lambda_{n}}(y)-\log P_{\Lambda_{n}}^{k}(y)\right| \leqq \sum_{t}\left|y_{t}-g_{k}\left(y_{t}\right)\right|,
$$

where the sum extends over all pairs of neighbours in $\Lambda_{n}$. Let $\tau$ denote the action of $Z^{d}$ on $Y, G_{k}(y)=\sum_{t, t \ni(0,0,0)}\left|y_{t}-g_{k}\left(y_{t}\right)\right|$.

We have then:

$$
\left|\log P_{\Lambda_{n}}(y)-\log P_{\Lambda_{n}}^{k}(y)\right| \leqq \sum_{\Lambda_{n}} G_{k}\left(\tau^{i} y\right)
$$


and

$$
s_{k}(y) \leqq \sup _{n} \frac{1}{n^{d}} \sum_{A_{n}} G_{k}\left(\tau^{i} y\right) .
$$

By a maximal ergodic lemma for a $Z^{d}$ action ([9], Theorem $I^{\prime}$ ), there exists a number $\lambda$ such that

$$
v\left\{\sup _{n} \frac{1}{n^{d}} \sum_{A_{n}} G_{k} \circ \tau^{i} \geqq \alpha\right\} \leqq \frac{\lambda}{\alpha} \int\left|G_{k}\right| d \nu \leqq \frac{\lambda}{\alpha} 2 d \delta_{k}
$$

and the lemma follows.

We can now proof Theorem 2. Let us choose a sequence $k_{i}$ such that $s_{k_{i}}(y)$ converges to zero almost everywhere. For almost every $y$, the conclusion of Lemma 8 holds for every $k_{i}$, we have $s_{k_{i}}(y) \rightarrow 0$ and

$$
\sup _{\substack{\mu \in M \\ \mu \circ \pi^{-1}=v}} \int a_{k_{i}} d \mu-\int a d \mu \rightarrow 0 \text { by Lemma } 9 .
$$

The conclusion of Theorem 2 follows.

Acknowledgements. I am grateful to Professors D. Ruelle and J. Lebowitz for discussions and particularly for suggesting the problem and to the referee P. A. Vuillermot for his remarks.

\section{References}

1. Ledrappier,F., Walters,P.: A relativised variational principle for continuous transformations. Preprint. To appear in J. London Math. Soc.

2. Rohlin,V.A.: Russ. Math. Surv. 22, 1-52 (1967)

3. Griffiths, R. B., Lebowitz,J.L.: J. Math. Phys. 9, 1284 (1968)

4. Gallavotti,G.: J. Math. Phys. 11, 141 (1972)

5. Essam, J. W.: In phase transition and critical phenomena (ed. C. Domb, Green). pp. 249-263. New York: Academic Press 1972

6. Edwards, S.F., Anderson,P.W.: J. Phys. F 5, 965 (1975)

7. Sherrington,D.: J. Phys. C: Solid St. Phys. 8, L 208 (1975)

8. Vuillermot, P.A.: Thermodynamics of quenched random spin systems and applications to the problem of phase transition in magnetic-(spin)-glasses. To appear in J. Phys. A. Math. Gen. (1977)

9. Wiener, N.: Duke Math. J. 5, 1-18 (1939)

Communicated by E. Lieb

Received March 18, 1977; in revised form July 11, 1977 